\title{
Dilemas morales y Derecho ${ }^{1}$
}

David Martínez Zorrilla²

${ }^{1}$ La realización de este artículo ha sido posible gracias a la ayuda de la Dirección General de Investigación del Ministerio de Educación y Ciencia de España a través del proyecto financiado SEJ2004-07136C02-01.

${ }^{2}$ Profesor ayudante de facultad (segundo período) de la Universidad Pompeu Fabra, Barcelona (España).
I.

El de los dilemas morales es sin ninguna duda uno de los temas más interesantes y al tiempo problemáticos del ámbito de la filosofía moral. La escasa atención $\mathrm{y}$ tratamiento que tradicionalmente recibieron los conflictos y dilemas, respecto de los cuales, por otra parte, predominaba la idea de que no había lugar para auténticos conflictos entre obligaciones morales (obligationes non colliduntur), contrasta claramente con el enorme interés que esta cuestión ha suscitado en las últimas 
décadas, en las que se han hecho muchas aportaciones significativas para entender mejor el problema ${ }^{3}$. En el ámbito de la teoría jurídica la situación ha sido distinta, puesto que, por una parte, los conflictos entre normas jurídicas (al menos en lo que al derecho positivo se refiere) se han concebido como situaciones relativamente frecuentes, o cuanto menos, no muy excepcionales, como lo muestra la larga tradición de los criterios para la resolución de antinomias (lex superior, lex posterior, etc. $)^{4}$, mientras que de otra parte apenas se ha prestado atención al impacto o relevancia que los conflictos y dilemas morales pueden tener en el ámbito jurídico.

En el presente trabajo no pretendo realizar un análisis detallado ni de los conflictos jurídicos ni de los dilemas morales, puesto que su pretensión es mucho más modesta y limitada: por una parte, se intentará ver bajo qué circunstancias una situación de dilema moral puede resultar jurídicamente relevante, o dicho en otros términos, cuándo puede ocurrir que el sistema jurídico, para dar respuesta a un caso, de algún modo remita al ámbito del discurso moral y en éste se plantee una situación de dilema; y por otra parte, qué consecuencias se pueden derivar de tales situaciones. Por otro lado, se trata de un trabajo de carácter predominantemente conceptual, en el que la pretensión principal (si bien no la exclusiva) es la clarificación de conceptos y de sus relaciones recíprocas. Por esta razón, más allá de consideraciones conceptuales generales, no centraré el interés en tratar de ver qué situaciones concretas, reales o hipotéticas, resultan o pueden resultar dilemáticas (sin que ello

\footnotetext{
${ }^{3}$ A modo de ejemplo, para una visión general de la situación, pueden verse las recopilaciones de artículos editadas por Gowans en GOWANS, C.W. (ed.) (1987), Moral Dilemas, Oxford, Oxford University Press; y Mason en MASON, H.E. (ed.) (1996), Moral Dilemas and Moral Theory, Oxford, Oxford University Press.

${ }^{4}$ Lo cual no impide que se hayan planteado importantes controversias acerca de si el sistema jurídico ofrece o no una única respuesta correcta para cada caso.
} 
impida que se ilustren algunas situaciones a modo de ejemplo). En la última parte del trabajo, en la sección VI, presentaré algunas propuestas muy limitadas de carácter normativo acerca de cómo dar respuesta a algunas situaciones de dilema, apuntando, como mera hipótesis, que posiblemente no sea adecuado en todos los casos resolver una controversia jurídica en la que un dilema moral es relevante del mismo modo que resolveríamos o responderíamos a la situación en el ámbito del discurso moral; en otras palabras: que aun cuando de algún modo el sistema jurídico nos remita al discurso moral y en éste se plantee una situación de dilema, que será entonces jurídicamente relevante, no necesariamente parecerá acertado resolverlo del mismo modo, debido a ciertas características importantes de los sistemas jurídicos.

El tema aquí tratado enlaza con muchas y muy complejas cuestiones tanto de la teoría y filosofía jurídicas como de la filosofía moral, que por razones de espacio no pueden ser tratadas detenidamente. Algunas de estas cuestiones han sido analizadas con mayor detalle y profundidad en otra parte ${ }^{5}$, y en aras de la brevedad, realizaré las remisiones oportunas cuando así lo requiera el hilo argumental. Antes de proseguir, quizá resulte adecuado señalar lo que no va a constituir el objeto del trabajo; esto es, situaciones que de algún modo pueden verse como conflictivas o dilemáticas y que afectan simultáneamente a los ámbitos moral y jurídico, pero que son distintas a lo que deseo analizar: por ejemplo, no me voy a interesar por las situaciones en las que el derecho ofrece una respuesta unívoca y determinada a un caso, mientras que la moral también ofrece su respuesta (asimismo unívoca y determinada), que es distinta e incompatible con la que ofrece el sistema jurídico, planteándose así un "dilema” entre las obligaciones moral y jurídica. De modo similar, tampoco prestaré atención

${ }^{5}$ En MARTÍNEZ ZORRILLA, D. (2004), Conflictos constitucionales, ponderación e indeterminación normativa (tesis doctoral inédita), Barcelona, UPF. 
a situaciones que siendo conflictivas en el ámbito jurídico (una antinomia jurídica), no lo son en el ámbito moral, o viceversa (situaciones de conflicto o dilema moral que cuentan con una solución unívoca y determinada en el derecho). Tampoco me interesan, por no constituir un verdadero 'dilema' según el concepto que más adelante se expondrá, las situaciones en que diversos sistemas morales alternativos ofrezcan soluciones divergentes e incompatibles, puesto que la noción de 'dilema' manejada es relativa a un único sistema normativo. Como se dijo al comienzo, el interés se centrará en aquellas situaciones en que el derecho de algún modo remita para la resolución del caso al razonamiento moral, y en éste se plantee una situación de dilema.

II.

Como punto de inicio, se partirá del concepto general de 'conflicto normativo', y se considerará que el de 'dilema' constituye una clase, definida por ciertas propiedades, dentro del concepto más amplio de 'conflicto normativo', que es relativo a un (cualquier) sistema normativo y por tanto común a los ámbitos jurídico y moral. Para la caracterización de ese concepto partiré de una noción intuitiva y pragmática, según la cual existe un conflicto normativo cada vez que el agente no puede satisfacer o cumplir todas las exigencias impuestas por el sistema normativo para ese caso (como ocurriría, citando un conocido ejemplo de Alchourrón ${ }^{6}$, si el sistema por una parte obliga al conductor a detenerse frente a un disco rojo, mientras que por otro lado prohíbe detenerse en zona militar, encontrándose el agente en zona militar frente a un disco rojo; en tal situación, haga lo que haga el agente, no satisfará todas las exigencias

\footnotetext{
${ }^{6}$ En ALCHOURRÓN, C.E. (1981), "G.H. von Wright y los desarrollos de la lógica deóntica”, en Anuario de Filosofía jurídica y social 1, p. 133.
} 
normativas del sistema para el caso). La razón por la que se parte de una noción puramente pragmática, en lugar de optar por definir los conflictos en términos lógicos (como inconsistencias lógicas del sistema normativo) es evitar tener que entrar en la compleja y discutida cuestión acerca de las relaciones entre los conflictos (en sentido pragmático) y la consistencia lógica del sistema ${ }^{7}$ (en otras palabras, si existe o no una relación de conexión necesaria y/o suficiente entre la existencia de inconsistencias lógicas en el sistema normativo y la aparición de situaciones de conflicto), que se suma al clásico problema de la existencia o no de relaciones lógicas entre normas.

La noción presentada de 'conflicto normativo' es, sin embargo, sólo embrionaria y requiere ser afinada y precisada, ya que presenta dos importantes dificultades: a) Por un lado, de acuerdo con el concepto presentado, una situación en la que sólo fuese aplicable al caso una única norma y por la razón que fuere el agente no pudiese cumplirla, sería calificada como un conflicto normativo, contrariamente al consenso generalizado; por el contrario, parece asumirse intuitivamente que para poder calificar una situación como de conflicto normativo ha de tratarse de situaciones en las que existen al menos dos normas simultáneamente aplicables, que el agente puede satisfacer o cumplir por separado, y que la imposibilidad de cumplimiento de alguna/s de ellas se debe precisamente al cumplimiento de la/s otra/s. b) Por otro lado, el concepto embrionario presentado no hace referencia alguna a las permisiones o facultamientos, lo que daría pie, por parte de algunos autores ${ }^{8}$, a negar la calificación de 'conflicto normativo' a situaciones como aquéllas en las que simultáneamente el sistema para un mismo caso prohíbe un comportamiento y permite o faculta un comportamiento incompatible (por

\footnotetext{
${ }^{7}$ Esta cuestión se analiza en MARTÍNEZ ZORRILLA, D. (2004), Conflictos... (cit.), cap. II. Secc. 4.

${ }^{8}$ Véase, por ejemplo, HERNÁNDEZ MARÍN, R. (1998), Introducción a la teoría de la norma jurídica, Madrid, Marcial Pons.
} 
ejemplo, una norma prohíbe fumar en la sala mientras que otra faculta hacerlo), ya sea con el argumento de que las permisiones no pueden ser cumplidas ni incumplidas, o con el argumento de que existe todavía un curso de acción en el que no se vulnera ninguna norma (en el ejemplo expuesto, abstenerse de fumar en la sala). Si queremos evitar tales dificultades, será necesario reformular el concepto de 'conflicto normativo', y mi propuesta es la siguiente: por 'conflicto normativo' se entiende cualquiera de las situaciones siguientes: a) toda situación en la que resulten aplicables al menos dos mandatos (obligaciones o prohibiciones), que el agente empíricamente puede cumplir individualmente, pero en la que el cumplimiento de cualquiera de ellos elimina la posibilidad empírica de cumplir con los demás; o b) toda situación en la que resulten aplicables al menos un mandato y un permiso, en la que el agente empíricamente puede cumplir el/los mandato/s y ejercitar el permiso individualmente, pero en la que el cumplimiento de algun/os mandato/s elimina la posibilidad empírica de ejercitar el permiso, o el ejercicio del permiso elimina la posibilidad empírica de cumplir con los demás mandatos ${ }^{9}$.

Por su parte, el concepto de 'dilema' (moral) parece ser más restringido. Por un lado, puede considerarse más restringido porque aunque resulta habitual su uso en el ámbito del discurso moral, no suele usarse en el ámbito jurídico (aunque no conozco ninguna buena razón para no aplicar este concepto al discurso jurídico), si bien en ocasiones se utiliza también en otros ámbitos del discurso práctico (como, por ejemplo, cuando se dice que alguien está frente a un dilema por no saber qué escoger entre dos destinos turísticos para sus vacaciones). Por otra parte, hasta donde llega mi conocimiento, no hay ningún autor que afirme la posibilidad de que surjan dilemas sin que exista al tiempo un conflicto normativo, aunque

${ }^{9}$ Se trata de la misma definición usada en MARTÍNEZ ZORRILLA, D. (2004), Conflictos... (cit.), p. 112. 
sí hay autores que tratan ambos conceptos como sinónimos o (la mayoría) que consideran los dilemas como una subcategoría específica dentro de la categoría más amplia de los conflictos morales. De cualquier modo, un aspecto destacable de la discusión filosófica acerca de los conflictos morales es que el discurso se ha centrado (en ocasiones de modo casi obsesivo) en la posibilidad de los dilemas, formando así dos "bandos": el de los autores que niegan toda posibilidad de que puedan plantearse dilemas (sosteniendo que todas las situaciones de conflicto son en último término aparentes y que hay una respuesta para el caso), y el de los autores que afirman la posibilidad real de su aparición ${ }^{10}$. Esta atención ha repercutido, en mi opinión, en un relativo descuido de los aspectos conceptuales, puesto que puede observarse, al menos en algunos casos, que la diferente posición frente a la posibilidad de que se planteen dilemas morales obedece a que se manejan (de manera no explícita, en muchos ocasiones) distintos conceptos de 'dilema', de modo que se plantea una falsa controversia, dado que en cierto sentido, ambos autores tienen razón. De cualquier modo, por el momento dejaremos de lado este concepto ${ }^{11}$ para centrar nuestra atención en el ámbito jurídico.

Como se ha afirmado anteriormente, para que una situación de dilema moral resulte jurídicamente relevante, el sistema jurídico debe de algún modo referirse o remitirse al discurso moral para la resolución del caso. De otro modo, lo que podemos tener es por ejemplo soluciones divergentes para un mismo caso según se analice éste desde la perspectiva jurídica o desde la perspectiva moral, o puede ocurrir que una situación sea moralmente dilemática pero que el sistema jurídico ofrezca una

\footnotetext{
${ }^{10}$ Citando algunos autores a modo de ejemplo: como contrarios a la posibilidad de los dilemas, encontramos a Earl Conee, Terrance McConnell o Alan Donagan; y como autores partidarios de su posibilidad pueden citarse entre otros a Bernard Williams, Ruth B. Marcus, Philippa Foot o Walter Sinnott-Armstrong.

${ }^{11}$ Se analizará en la sección IV.
} 
respuesta determinada y unívoca, en cuyo caso, desde la perspectiva estrictamente jurídica, el dilema moral sería irrelevante.

Una de las posibilidades existentes para que se dé esta conexión y un dilema pueda llegar a tener relevancia jurídica se plantea en aquellas normas jurídicas en las que para la solución del caso se hace una remisión a la moral o se utilizan conceptos propios del discurso moral, que hacen necesario un razonamiento de carácter moral para hallar o establecer (para ser neutrales desde un punto de vista metaético) la solución del caso. En el derecho español podemos hallar varios ejemplos de este tipo de remisiones, siendo posiblemente el más conocido el del artículo $1255 \mathrm{del}$ Código Civil, que establece literalmente: "Los contratantes pueden establecer los pactos, cláusulas y condiciones que tengan por convenientes, siempre que no sean contrarios a las leyes, a la moral, ni al orden público". Dicho precepto (en combinación con otros) establece como condición de la validez contractual la de su corrección moral, por lo que en la hipótesis de que desde una perspectiva moral se plantease un dilema (por ejemplo, por poder ampararse el objeto del contrato en un principio moral al tiempo que resulte contrario a otro principio), dicho dilema pasaría a ser jurídicamente relevante.

No son estas situaciones, sin embargo, las que en mi opinión plantean el vínculo más estrecho entre los dilemas morales y el derecho. Dicho vínculo se manifiesta más claramente, a mi parecer, en las situaciones que pueden calificarse como 'conflictos constitucionales' ${ }^{12}$. Con ello no

\footnotetext{
${ }^{12}$ En realidad, este vínculo entre el derecho y los dilemas morales puede plantearse también en cualquier situación de indeterminación normativa debida a un problema de laguna normativa o de antinomia. En esas situaciones, como (desde la perspectiva positivista hartiana) el decisor goza de discrecionalidad, puede acudir al razonamiento moral para tomar su decisión, y podría ocurrir que en el ámbito moral se plantease un dilema. En cualquier caso, no incluyo estas situaciones porque no hay por qué pensar que necesariamente (o incluso
} 
pretendo sostener ni que toda situación de conflicto constitucional dé lugar a un dilema moral, ni tampoco que los dilemas morales sólo puedan tener relevancia jurídica a través de los conflictos constitucionales (como lo muestra el ejemplo del art. 1255 del Código Civil español). Mi hipótesis es simplemente que la mayoría de situaciones en las que un dilema moral tenga relevancia jurídica, serán situaciones de conflicto constitucional.

\section{III.}

Para una adecuada comprensión de estas situaciones que se han denominado 'conflictos constitucionales', así como de las consecuencias teóricas y prácticas que conllevan, conviene tener en cuenta que nos hallamos inmersos en un contexto que muchos autores han denominado como 'neoconstitucionalismo'. Aunque resulta complicado precisar qué propiedades o características definen este contexto ${ }^{13}$, a efectos de lo que aquí interesa basta con señalar lo siguiente: Si durante el siglo XIX y primeras décadas del XX los textos constitucionales occidentales fundamentalmente abarcaban y regulaban aspectos institucionales y procedimentales (tales como la estructura jurídico-política básica del estado, la composición y funciones de sus principales órganos, los procedimientos legislativos, etc.), en tiempos más recientes, fundamentalmente durante el último medio siglo, se han ido incorporando otros elementos de marcado contenido sustantivo, usualmente denominados como 'derechos

usualmente) se trate de controversias que tengan relevancia moral o que deban ser resueltas conforme a un razonamiento de tipo moral (caben muchas otras alternativas, como usar criterios económicos, de oportunidad, políticos, etc.), mientras que la vinculación con el discurso moral es mucho más estrecha en el caso de los conflictos constitucionales.

${ }^{13}$ Una de las exposiciones más completas y sistemáticas de las características que definirían un contexto jurídico neoconstitucionalista puede encontrarse en GUASTINI, R. (2003), "La constitucionalización del ordenamiento jurídico", en CARBONELL, M. (ed.), Neoconstitucionalismo(s), Madrid, Trotta, pp. 49-73. 
fundamentales', 'bienes constitucionales, 'valores', 'principios', etc. Dichos elementos suelen ser expresados en un lenguaje con un alto nivel de vaguedad y una evidente carga emotiva, lo cual ha provocado, junto con la consideración de las constituciones (incluyendo todos sus preceptos) como auténticas normas jurídicas vinculantes y superiores a cualquier otra fuente, que un gran número de controversias jurídicas tengan "relevancia constitucional", en el sentido de que resulta muy habitual que una pretensión pueda entenderse como que está jurídicamente amparada por alguno de estos derechos, valores, bienes o principios constitucionales. Lo anterior además se complica por el hecho de que, al estar expresados en términos tan generales, vagos y emotivos, en muchas ocasiones las pretensiones opuestas en un conflicto pueden entenderse, al menos prima facie, como que ambas se encuentran constitucionalmente amparadas, al encontrar acomodo dentro del gran elenco de derechos, principios, bienes y valores reconocidos por las constituciones.

Tales situaciones en las que pretensiones distintas e incompatibles pueden verse (o al menos así se presentan) como simultáneamente protegidas por derechos, bienes, etc. constitucionales son las que aquí se denominan genéricamente como 'conflictos constitucionales'. A modo de ejemplo, en la jurisprudencia constitucional española, una situación bastante habitual ha sido aquélla en la que la publicación de cierta noticia o información en un medio de comunicación repercute negativamente, por su contenido, en la credibilidad, fama o buena imagen pública de otra u otras personas, o bien afecta a su intimidad. Sin entrar en más detalles y en una primera aproximación, en situaciones de este tipo la pretensión del periodista de hacer pública la noticia o dar esa información encuentra amparo en lo dispuesto por el art. 20.1 d) de la constitución española ${ }^{14}$, mientras que la pretensión de las personas afectadas de que su imagen no

\footnotetext{
${ }^{14}$ Art. 20.1 d) CE: "Se reconocen y protegen los derechos: (...) d) A comunicar o recibir libremente información veraz por cualquier medio de difusión (...)".
} 
se vea perjudicada o su intimidad puesta al descubierto hallaría amparo en el art. 18.1 de la misma ${ }^{15}$.

Las situaciones de conflicto constitucional, al margen de las evidentes dificultades prácticas que plantean (pues los tribunales han de dar respuesta a estos casos), suscitan también complejas e interesantes cuestiones teóricas, como por ejemplo: a) qué tipo de normas jurídicas son las que entran en juego (es común afirmar que los preceptos que entran en colisión son principios, en contraposición a las reglas); b) problemas de interpretación normativa (si se admite la distinción entre 'disposición normativa' -como enunciado linguiístico- y 'norma' -como significado de la disposición, obtenido mediante la interpretación-, es claro que podría darse el caso de que en algunas interpretaciones, el conflicto podría manifestarse, mientras que en otras no, y la cuestión es problemática debido al alto nivel de vaguedad y carga emotiva de las expresiones utilizadas; c) calificación de estas situaciones, siguiendo la terminología de Guastini ${ }^{16}$, como 'conflictos in concreto' o como 'conflictos in abstracto'; d) carácter "real" o meramente aparente de estos conflictos; etc. Algunas de estas cuestiones ya han sido analizadas con detenimiento en otro lugar ${ }^{17}$, por lo que no voy a entrar en ellas. Se partirá, eso sí, de la idea de que al menos algunas de estas situaciones son de auténtico conflicto, es decir, que se ajustan a la definición de 'conflicto normativo' expuesta en II, a pesar de que no faltan autores que consideran que se trata siempre de aparentes conflictos ${ }^{18}$.

\footnotetext{
${ }^{15}$ Art. 18.1 CE: "Se garantiza el derecho al honor, a la intimidad personal y familiar, y a la propia imagen".

${ }^{16}$ Esta distinción se puede hallar en GUASTINI, R. (1996), "I principi nel diritto positivo", en Distinguendo, Torino. Giappichelli.

${ }^{17}$ En MARTÍNEZ ZORRILLA, D. (2004), Conflictos... (cit.), cap. II.

${ }^{18}$ Entre otros, es el caso de Ignacio de Otto en De OTTO, I. (1998), "La regulación del ejercicio de los derechos y libertades. La garantía de su contenido esencial en el artículo 53.3 de la Constitución”, en De OTTO, I., y MARTÍN-RETORTILLO, L. (1988), Derechos
} 
Un aspecto destacable de las situaciones de conflicto constitucional es que no se les puede hacer frente, para su resolución, mediante los clásicos criterios de resolución de antinomias jurídicas. No puede usarse el criterio de lex superior, porque se asume (como regla general, al menos) que todos los preceptos constitucionales tienen la misma jerarquía formal ${ }^{19}$; tampoco el de lex posterior, por formar parte de un único texto legal y coetáneo. No existiría impedimento, como creo que adecuadamente sostiene Guastini ${ }^{20}$, para la posibilidad de usar el criterio de lex specialis, aunque ello habitualmente no será posible porque no suelen plantearse las relaciones de especialidad requeridas para hacer uso de este criterio. Por ello, es prácticamente unánime la idea de que se requiere otro mecanismo distinto y específico para dar respuesta y solución a los conflictos constitucionales, y a tal mecanismo se le suele denominar 'ponderación' ${ }^{21}$. Desgraciadamente, ocurre que el de 'ponderación' es uno de los conceptos más oscuros, resbaladizos y problemáticos de toda la teoría jurídica, y

fundamentales y constitución, Madrid, Tecnos. También JIMÉNEZ CAMPO, J. (1999), Derechos fundamentales. Concepto y garantías, Madrid, Trotta. En otro sentido, SERNA. P. y TOLLER, F. (2000), La interpretación constitucional de los derechos fundamentales. Una alternativa a los conflictos de derechos, Buenos Aires, La Ley. En la misma línea, CIANCIARDO, J. (2000), El conflictivismo en los derechos fundamentales, Pamplona, EUNSA; así como OLLERO, A. (2000), "La ponderación delimitadora de los derechos humanos: libertad informativa e intimidad personal", en Pensamiento y cultura 3, pp. 157-166.

${ }^{19}$ Más discutido y problemático es el establecimiento de jerarquías materiales que en ocasiones se ha propuesto por parte de algunos autores u órganos judiciales.

${ }^{20} \mathrm{Vid}$. GUASTINI, R. (1998), "Principi di diritto e discrecionalita giudiziale", en Diritto Pubblico 3, pp. 641-660.

${ }^{21}$ Hay que tener en cuenta, no obstante, que no sólo la ponderación no es el único instrumento conceptualmente posible para la resolución de estos conflictos, sino que además, de hecho, se han planteado alternativas muy interesantes, como la teoría coherentista de Susan Hurley, en HURLEY, S.L. (1989), Natural Reasons. Personality and Polity, New York, Oxford University Press, y HURLEY, S.L. (1990), "Coherence, Hypothetical Cases and Precedent”, en Oxford Journal of Legal Studies 1990 pp. 221-251. 
por razones de espacio no podemos tratarlo en detalle ${ }^{22}$. Lo único que voy a destacar de la ponderación es que ésta puede analizarse, al menos, desde las tres perspectivas siguientes: a) conceptual (en qué consiste la ponderación), b) metodológica (cómo se lleva a cabo la ponderación), y c) normativa (cómo se debe ponderar, o cuándo y cómo una ponderación puede considerarse correcta o justificada). Por desgracia, estos tres aspectos o perspectivas de análisis no suelen ser convenientemente distinguidos, y en ocasiones se entremezclan. Además, si bien, por lo que respecta al aspecto conceptual, podría decirse que, al margen de destacables diferencias entre autores, existiría un consenso sobre el núcleo del concepto (según el cual la ponderación consistiría en la atribución o establecimiento de un valor a cada una de las alternativas en conflicto, conforme al cual se otorga preferencia a la alternativa de mayor valor), las diferencias en los otros dos ámbitos son más importantes. La falta de distinción adecuada entre estas distintas dimensiones de la ponderación se manifiesta incluso en autores reconocidos que han dedicado importantes esfuerzos teóricos a la ponderación, como es el caso de Robert Alexy. Alexy se refiere a la ponderación desde una perspectiva metodológica cuando sostiene que la ponderación se realiza mediante el establecimiento de una relación de precedencia condicionada entre los dos principios en conflicto, de tal modo que dicha relación da lugar a una regla con la estructura ' $\mathrm{C} \rightarrow \mathrm{R}$ ', en donde ' $\mathrm{C}$ ' son las circunstancias de la relación de precedencia, que actúan como supuesto de hecho de la regla, y ' $R$ ' es la consecuencia jurídica prescrita por el principio que tiene preferencia ${ }^{2324}$. En cambio, un poco más

${ }^{22}$ Un análisis detallado se encuentra en MARTÍNEZZZORILLA, D. (2004), Conflictos... (cit.), cap. III.

${ }^{23}$ Vid. ALEXY, R. (1993), Teoría de los derechos fundamentales, Madrid, CEC, pp. 90-98.

${ }^{24}$ La concepción de la ponderación, desde esta perspectiva metodológica, como un mecanismo para la elaboración de reglas que permitan resolver futuros casos mediante un razonamiento subsuntivo es también mantenida por otros autores como Prieto (por ejemplo, en PRIETO, L. (2003), “El juicio de ponderación”, en Justicia constitucional y derechos 
adelante $^{25}$ el autor se sitúa en una perspectiva normativa cuando sostiene que la ponderación se rige por la ‘ley de la ponderación' o ‘principio de proporcionalidad' (subdividido a su vez en los subprincipios de adecuación o idoneidad, de necesidad y de proporcionalidad en sentido estricto), conforme al cual, en síntesis, cuanto mayor es el grado de afectación o no satisfacción de uno de los principios en juego, tanto mayor debe ser el grado de satisfacción o realización del otro. Esto es, de lo que ahora se trata es de tener un criterio o procedimiento que determine cuándo una ponderación es correcta o está justificada desde una perspectiva axiológica; en definitiva, cómo hay que regirse a la hora de establecer preferencias condicionadas (en forma de reglas) entre los elementos en conflicto. Lo que en mi opinión es un error de Alexy es el pensar que existe una vinculación conceptual entre el establecimiento de relaciones de precedencia condicionada y el principio de proporcionalidad, cuando no existe impedimento conceptual alguno en imaginar algún mecanismo o criterio para el establecimiento de relaciones de precedencia condicionada basados en algo distinto a los grados de afectación o satisfacción de los elementos en conflicto.

De las tres perspectivas señaladas, me interesa ahora la normativa, por lo siguiente: como se ha dicho reiteradamente, los elementos (derechos, bienes, valores, principios, etc.) que entran en juego en las situaciones de conflicto constitucional cuentan con una importante carga valorativa, y muchos de ellos pueden ser considerados netamente como conceptos

fundamentales, Madrid, Trotta, pp. 175-216), Moreso (en MORESO, J.J. (2003), "Conflictos entre principios constitucionales", en CARBONELL, M. (ed.), Neoconstitucionalismo(s), Madrid, Trotta, pp. 99-121), o Mendonca (en MENDOCA, D. (2003), Los derechos en juego. Conflicto y balance de derechos, Madrid, Tecnos), y se trata de una concepción muy atractiva a la cual me adhiero en MARTÍNEZ ZORRILLA, D. (2004), Conflictos... (cit.), cap. III.

${ }^{25}$ ALEXY, R. (1993), Teoría... (cit.), pp. 161 ss. 
morales o propios del discurso moral (por ejemplo, y al margen del propio concepto de 'derecho humano', o 'derecho fundamental', aparecen otros como 'dignidad humana', 'tratos inhumanos o degradantes', 'integridad moral', 'honor', 'libre desarrollo de la personalidad', 'justicia', etc. ${ }^{26}$ ). Dado que todos ellos forman parte de igual modo del texto constitucional y carecemos, en principio, de criterios estrictamente formales (como lex superior, etc.) para determinar una solución, parece que la alternativa más razonable es trasladar la cuestión al discurso moral ${ }^{27}$, a fin de poder ofrecer también una respuesta a la controversia jurídica, que en su contenido tampoco difiere de manera destacable de una controversia moral.

En este contexto, en la hipótesis de que en el ámbito moral se plantee un dilema, de modo que no pueda determinarse qué alternativa debe escogerse o es la correcta, este dilema se trasladará asimismo al ámbito jurídico; o, dicho de otra manera, será un dilema moral relevante jurídicamente. Es por esta razón por la que antes se ha considerado que el de los conflictos constitucionales es probablemente el vínculo más destacable entre los dilemas morales y el ámbito jurídico. Dicho esto, es el momento de entrar en mayores detalles por lo que respecta a los dilemas morales.

\section{IV.}

Como se indicó anteriormente, el debate filosófico sobre los dilemas morales ha puesto el acento en la discusión acerca de su posibilidad (tanto conceptual como empírica), esto es, si pueden o no plantearse auténticas situaciones de dilema. En cambio, en ocasiones lo que ha ocurrido es que

\footnotetext{
${ }^{26}$ Todos estos conceptos aparecen en el texto de la constitución española de 1978.

${ }^{27}$ Nótese que se dice "alternativa más razonable" y no "la única" o "la única posible", ya que podría solucionarse la cuestión de otro modo, por ejemplo, mediante criterios económicos de cálculo coste-beneficio.
} 
el debate ha resultado un tanto artificial, porque el concepto de 'dilema' manejado no era siempre coincidente entre los diversos autores y ello determinaba en parte su posición acerca de la posibilidad de los dilemas. Por ello, considero que es de gran importancia contar con un concepto claramente determinado de 'dilema', aun a costa de que pueda resultar un tanto estipulativo, para clarificar el análisis y poder plantear la discusión en términos más claros.

En primer lugar, parece claro que cuando hablamos de dilemas (morales) estamos hablando de algo emparentado con los conflictos normativos (morales). No conozco ningún autor que no considere que un dilema es al mismo tiempo una situación de conflicto. Ahora bien, mientras que hay algunos que tratan los términos 'conflicto' y 'dilema' como sinónimos ${ }^{28}$, la mayoría parece restringir la noción de 'dilema' a una categoría específica de conflicto, normalmente a un tipo de conflicto normativo que resulta especialmente "importante" o "problemático". Lo que ocurre es que hay diversos modos de considerar que un conflicto es "problemático" o "importante". Una posibilidad es atender al grado de importancia o relevancia que, desde un punto de vista valorativo (no necesariamente moral), tienen los derechos, intereses, bienes o valores en juego. Desde este punto de vista, no sería merecedora de llamarse 'dilema' una situación de conflicto entre dos promesas de contenido trivial, mientras que sí lo sería (o podría serlo) la situación en la que se ve involucrada la vida de las personas (por ejemplo, por tener que decidir a quién se salva la vida, siendo imposible salvársela a todos). La principal dificultad que ofrece este criterio es que resulta demasiado vago y subjetivo, de modo que no

${ }^{28}$ Tal sería el caso de Alan Donagan, en DONAGAN, A. (1977), The Theory of Morality, Chicago, Chicago University Press, y en DONAGAN, A. (1996), "Moral Dilemas, Genuine and Spurious: A Comparative Anatomy", en MASON, H.E. (ed.), Moral Dilemas and Moral Theory, Oxford, Oxford University Press, pp. 11-22. 
siempre se podrá distinguir claramente una situación de dilema moral de otra de "simple" conflicto. Por esta razón, resulta preferible seguir otra estrategia, y esa estrategia consiste en hacer depender el concepto de la estructura del conflicto normativo. De ese modo, los dilemas morales serán aquellos conflictos normativos (morales) que presenten cierta estructura, que no presentan siempre los conflictos normativos.

Resulta bastante habitual en la literatura sobre conflictos y dilemas morales diferenciar entre las obligaciones prima facie y las obligaciones 'all-things considered' (que aquí podríamos traducir como 'obligaciones definitivas'). La noción de obligación prima facie fue introducida por W.D. Ross en $1930^{29}$ y desde entonces su uso se ha extendido de manera considerable. Resulta necesario, no obstante, aclarar que la expresión 'obligación prima facie' es ambigua, puesto que se utiliza en dos sentidos distintos, a saber:

a)

Según la posición que ocupa en el razonamiento relativo a qué se debe hacer. En este sentido, que es el que predomina en la filosofía moral, es un deber u obligación prima facie toda aquella alternativa de acción en un conflicto, que en principio o a primera vista (prima facie) debe llevarse a cabo, pero que resulta finalmente desechada o superada por otra alternativa que se juzga como superior o más importante, que es lo que en definitiva debe hacerse (obligación definitiva).

b) Como sinónimo de 'derrotable’ (defeasible). La idea de 'derrotabilidad', que tiene su origen en el desarrollo de las lógicas no monótonas pero que ha visto

\footnotetext{
${ }^{29}$ En ROSS, W.D. (1930), The Right and the Good, Oxford, Clarendon Press.
} 
ampliada su aplicación a muchos otros campos, supone, en síntesis, y aplicada al ámbito normativo, que para la aplicación de una norma es necesario, además de la concurrencia de todas las circunstancias establecidas en su antecedente, todo un conjunto de otras circunstancias implícitas que en el supuesto de no concurrir provocan que la norma sea 'derrotada', y en consecuencia no aplicable. Usando un ejemplo del ámbito jurídico, la obligación del juez de castigar al homicida es una obligación derrotable porque no basta con que se haya dado la circunstancia de que alguien haya matado a otro, sino además es necesario que concurran otros requisitos (que el homicida sea mayor de edad penal, que no se hallara en situación de enajenación mental, que no fuera un acto de legítima defensa, etc.) que en caso de no concurrir provocan que la norma no pueda aplicarse.

Aunque relacionados, se trata de dos sentidos distintos, puesto que si bien necesariamente toda obligación prima facie en el primer sentido es asimismo una obligación derrotable (y derrotada), no ocurre a la inversa: una obligación puede ser derrotable y no ser, en algunos casos, una obligación prima facie en el primer sentido, sino una obligación definitiva (por ejemplo, cuando no entra en colisión con otras obligaciones, o cuando a pesar del conflicto, resulte la obligación vencedora).

Para el propósito de este trabajo, se entenderá como 'dilema (moral)' toda situación de conflicto normativo (moral) en la que el sistema normativo (moral) carezca de recursos (criterios, escalas, procedimientos, etc.) para poder determinar la obligación definitiva de entre las distintas obligaciones prima facie en conflicto; esto es, serán dilemáticas aquellas situaciones de conflicto en las que no pueda 
establecerse la obligación definitiva. Se trata de un criterio netamente estructural que no entra a considerar la "importancia" de la situación ni los valores, derechos o bienes involucrados en ella, de modo que puede darse perfectamente el caso de que un conflicto entre dos obligaciones triviales resulte ser un dilema, mientras que otra situación en la que los bienes en juego son más importantes y cualquier alternativa desagradable (como por ejemplo tener que escoger entre salvar a un paciente a costa de provocarle graves disfunciones físicas y psicológicas o dejarlo morir) puede no serlo.

v.

No es el propósito del presente trabajo decir si en la práctica (en lo que llamaríamos "el mundo real") estas situaciones son posibles o si por el contrario nunca se manifiestan, o cuáles serían ejemplos concretos de situaciones de dilema. Incluso no se niega la posibilidad de que en algunos sistemas morales (quizás en el sistema moral "verdadero" de los objetivistas cognoscitivistas) no quepa la posibilidad de indeterminación que caracteriza a los dilemas. Si son posibles, y en qué casos pueden surgir dilemas morales, es algo que dependerá del concreto contenido que tenga el sistema normativo moral manejado. El único propósito aquí es conceptual: si ocurren situaciones de conflicto normativo en el ámbito moral en las que el sistema no permita determinar cuál es la obligación definitiva de entre las distintas obligaciones prima facie en conflicto, estaremos frente a un dilema moral. De lo que ahora se trata es de ver en qué situaciones los dilemas serían conceptualmente posibles (independientemente de si se manifiestan o no en la práctica) y cuáles serían las consecuencias normativas de tales situaciones. Bajo mi punto de vista, existen dos claros candidatos para situaciones de dilema: a) la equivalencia entre las alternativas; b) los casos de incomparabilidad. 


\section{La equivalencia de las alternativas}

Simplificando los términos, diríamos que las situaciones de equivalencia son aquellas en las que las obligaciones incompatibles en disputa "valen" lo mismo, en el sentido de que su valor o importancia desde el punto de vista deóntico es idéntico, de manera que no puede reputarse a una de las alternativas como de mayor importancia que la/s otra/s, y ninguna obligación desplaza a las demás convirtiéndose así en la obligación definitiva. En pocas palabras, serían casos de empate. Las situaciones de equivalencia requieren conceptualmente que las alternativas en conflicto sean comparables y conmensurables (evaluables en términos de una escala común a la que se pueden reducir o conforme a la cual se pueden evaluar), y esto las diferenciaría de las situaciones de incomparabilidad que veremos más adelante.

Es importante destacar que para que un conflicto normativo constituya una auténtica equivalencia entre alternativas, éstas últimas han de ser iguales en su valor deóntico desde todos los puntos de vista moralmente relevantes (cuáles sean éstos es algo que dependerá del sistema moral manejado). Como resulta habitual que muchas situaciones o cursos de acción sean relevantes desde distintas perspectivas o atendiendo a distintos criterios moralmente relevantes, es razonable pensar que los casos de auténtica equivalencia, aunque conceptualmente posibles, resultarán poco frecuentes. Por ejemplo: imaginemos una situación en la que el agente no puede satisfacer dos promesas que hizo porque el cumplimiento de cualquiera de ellas impide el de la otra. Los comportamientos en juego, en tanto que cumplimiento de promesas, tienen el mismo valor deóntico, porque ambos se subsumen en el principio moral general de que deben cumplirse las promesas. Ahora bien, puede perfectamente ocurrir que desde otros puntos de vista moralmente relevantes (por ejemplo, cómo quedan afectados en cada caso los intereses de la otra parte y de terceros en los supuestos de cumplimiento o incumplimiento), las alternativas resulten muy distintas, lo que constituiría un criterio para decidir qué 
alternativa ejecutar. Incluso en situaciones que parecen muy equilibradas, como podría ser aquélla en la que debe decidirse a quién, de entre dos pacientes, se le aplica un tratamiento médico para salvarle la vida, no habiendo recursos suficientes para tratar a los dos pacientes, pueden existir diferencias en aspectos moralmente relevantes que determinen la solución del conflicto (por ejemplo, podría ocurrir que la vida de un paciente sólo pudiera ser salvada a costa de graves dolores y limitaciones, mientras que el otro paciente llevaría una vida normal, o que uno de los pacientes tenga unas probabilidades de éxito en el tratamiento significativamente mayores, o que uno de los pacientes sea significativamente más joven, o que requiera de menos recursos para su tratamiento, de modo que podrían ser tratadas más personas, etc.). Quizás un supuesto de auténtica equivalencia sería el de dos siameses que comparten varios órganos vitales, de modo que sólo puede sobrevivir uno de ellos, siendo las posibilidades de éxito similares para cualquiera de los dos.

Pero dejando al margen el tema de la posibilidad "real" de que se planteen casos de equivalencia, si así fuera estaríamos ante una situación de dilema. La cuestión es ahora qué consecuencias se derivarían de dicha situación. Una posibilidad es pensar que cualquier decisión que se tome estará igualmente injustificada y será por ello incorrecta, de modo que no es posible evitar el "mal" o el "error" (y éste sería uno de los elementos trágicos de las situaciones de dilema). Otra alternativa, muy interesante, es la que adoptan autores como A. Donagan ${ }^{30}$, E. Conee ${ }^{31}$, T. McConnell ${ }^{32}$,

${ }^{30}$ Vid. DONAGAN, A. (1984), "Consistency in Racional Moral Systems”, en GOWANS, C.W. (ed.) (1987), Moral... (cit.), pp. 271-290.

${ }^{31}$ Vid. CONEE, E. (1982), “Against Moral Dilemmas”, en GOWANS, C.W. (ed.) (1987), Moral... (cit.), pp. 239-249.

${ }^{32}$ Vid. McCONNELL, T. (1993), "Dilemmas and Incommensurabilities", en The Journal of Value Enquiry 27, pp. 247-252. 
D. Brink ${ }^{33}$ o M.J. Zimmerman ${ }^{34}$. Estos autores, en lugar de sostener que cualquier decisión que se tome será incorrecta, afirman que todas las alternativas en conflicto están moralmente permitidas, o dicho de manera más precisa, que existiría una obligación alternativa entre las distintas obligaciones prima facie, de modo que debe cumplirse alguna de las obligaciones en conflicto, pero no importa, desde el punto de vista moral, cuál de ellas se lleve a cabo. Lo único que no sería moralmente correcto sería eludir todas las obligaciones en conflicto, pero cumpliendo cualquiera de ellas, sin importar cuál, nuestro comportamiento sería moralmente irreprochable. También Alexy, en el ámbito de la teoría jurídica, mantiene una posición muy similar, afirmando que en los casos de empate existe discrecionalidad ${ }^{35}$.

${ }^{33}$ Vid. BRINK, D.O. (1996), "Moral Conflict and its Structure”, en MASON, H.E. (ed.), Moral... (cit.), pp. 102-126.

34 Vid. ZIMMERMAN, M.J. (1996), The Concept of Moral Obligation, Cambridge, Cambridge University Press.

${ }^{35}$ ALEXY, R. (2002), "Epílogo a la Teoría de los derechos fundamentales", en Revista Española de Derecho Constitucional, nº 66, pp. 13-64; ALEXY, R. (2003a), "Constitucional Rights, Balancing, and Rationality”, en Ratio Iuris vol. 16 n² 2, pp. 131-140; y ALEXY, R. (2003b), On Balancing and Subsumption. A Structural Comparison", en Ratio Iuris vol. 16 $n^{\circ} 4$, pp. 433-449. En su desarrollo del principio de proporcionalidad que debe regir la ponderación, este autor propone una escala triádica conforme a la cual puede valorarse tanto el grado o nivel de afectación o lesión de uno de los principios en conflicto, como el grado o nivel de satisfacción o realización del otro, según cuál sea la alternativa a elegir. Esta afectación o satisfacción puede ser 'leve', 'moderada' o 'grave' (o 'intensa' si hablamos de satisfacción y no de lesión), y el principio de proporcionalidad supone una preferencia de una alternativa sobre otra en función de los grados que han sido asignados a cada elemento (por ejemplo, si una alternativa supone una satisfacción 'intensa' de un principio mientras que supone sólo una lesión 'leve' del otro, ésa sería la alternativa correcta. No obstante, existen tres casos de empate: aquellos en que tanto la afectación como la satisfacción son ambos 'leves', 'moderados' o ‘intensos'. En tales casos, dice Alexy, existe discrecionalidad, que se traduce, en el contexto del examen de constitucionalidad que llevan a cabo determinados órganos judiciales, en que la ley debe ser declarada constitucional, pues al haber discrecionalidad corresponde al legislativo decidir libremente cómo regular la cuestión. 
A fin de analizar si este punto de vista es correcto, deberíamos tratar de ver si se trata de una tesis normativa, que se limita a prescribir cómo hemos de actuar frente a situaciones de equivalencia, o bien si se trata de una tesis conceptual, esto es, de algo que se sigue lógica o conceptualmente a partir de los propios conceptos de 'obligación moral' y 'permisión moral'. La primera alternativa es menos interesante desde el punto de vista filosófico, puesto que se trataría de algo contingente, relativo al sistema normativo manejado, que puede contener o no una prescripción de este tipo para guiar la conducta en casos de equivalencia. Por el contrario, si se trata de una tesis conceptual, podremos afirmar que en ese tipo de supuestos necesariamente existe una obligación de realizar alguno de los comportamientos incompatibles, sin que exista ninguna preferencia por alguno de ellos puesto que ambos están moralmente permitidos.

Para analizar la cuestión desde esta perspectiva conceptual, partiremos del examen del concepto de 'obligación moral' desarrollado por M. J. Zimmerman ${ }^{36}$. Conforme a este autor, que algo es moralmente obligatorio significa: el agente $S$ debe, en el momento T, hacer A en T', si y sólo si a) S puede (empíricamente) hacer $A$ en $T^{\prime}$; b) $S$ puede (empíricamente) abstenerse de hacer $A$ en $T$; ; y c) todo mundo accesible en el que $S$ no hace A en T' es deónticamente inferior a algún mundo en

${ }^{36}$ En ZIMMERMAN, M.J. (1996), The Concept... (cit.). El análisis de este autor plantea complejas e interesantes cuestiones que no pueden ser aquí discutidas, como por ejemplo hasta qué punto sería su reconstrucción compatible con las diferentes concepciones metaéticas, o si su análisis de expresiones como 'debo moralmente hacer A' se refiere a genuinas normas o bien a proposiciones normativas. Para algunos comentarios sobre estas cuestiones, vid. MARTÍNEZ ZORRILLA, D. (2004), Conflictos... (cit.), cap. IV sec 1.1. Bajo mi punto de vista, la reconstrucción de Zimmerman resulta neutral en relación con las distintas concepciones metaéticas y se refiere a proposiciones normativas. 
el que S hace A en $T^{37}$. Por su parte, la permisión moral se define en los términos siguientes: el agente $S$ puede (deónticamente), en el momento T, hacer A en T', si y sólo si a) S puede (empíricamente) hacer A en T'; b) S puede (empíricamente) abstenerse de hacer $A$ en $T$ '; y c) cualquier mundo accesible en el que $S$ no hace $A$ en $T$ ' no es deónticamente superior a algún mundo accesible en el que $S$ hace A en $T^{\prime 38}$.

${ }^{37}$ Vid. ZIMMERMAN, M.J. (1996), The Concept... (cit.), p. 26. En pp. 26 y 27 ofrece la siguiente definición, más formalizada:

$\mathrm{El}$ agente $\mathrm{S}$ debe, en el momento T en el mundo W, hacer A en T', si y sólo si:

a) Existe un mundo $\mathrm{W}^{\prime}$ tal que $\mathrm{W}^{\prime}$ es accesible para $\mathrm{S}$ desde $\mathrm{W}$ en $\mathrm{T}$ y $\mathrm{S}$ hace A en T' en W'

b) Existe un mundo W" tal que W" es accesible para $\mathrm{S}$ desde $\mathrm{W}$ en $\mathrm{T}$ y $\mathrm{S}$ no hace A en T' en W'

c) Para todo mundo W" tal que W" es accesible para A desde W en T y S no hace A en T' en W', existe un mundo W' tal que:

1) $W^{\prime}$ es accesible para $S$ en $W$ en $T$

2) S hace A en T' en W'

3) El valor deóntico para $\mathrm{S}$ en $\mathrm{T}$ de W' es mayor que el valor deóntico para S en T de W", y

4) No existe ningún mundo $W$ '" tal que

i) W'" es accesible para $\mathrm{S}$ desde $\mathrm{W}$ en $\mathrm{T}$

ii) S no hace A en T' en W',', y

iii) El valor deóntico para $\mathrm{S}$ en $\mathrm{T}$ de $\mathrm{W}$ '” es mayor que el valor deóntico para $\mathrm{S}$ en $\mathrm{T}$ de $\mathrm{W}$ '

${ }^{38}$ Vid. ZIMMERMAN, M.J. (1996), The Concept... (cit.), p. 32. En una versión más formalizada (p.32):

El agente S puede (deónticamente), en T en W, hacer A en T' si y sólo si:

a) Existe un mundo $\mathrm{W}^{\prime}$ tal que $\mathrm{W}^{\prime}$ ' es accesible para $\mathrm{S}$ desde $\mathrm{W}$ en $\mathrm{T}$ y $\mathrm{S}$ hace A en T' en W'

b) Existe un mundo W" tal que W" es accesible para $\mathrm{S}$ desde $\mathrm{W}$ en $\mathrm{T}$ y $\mathrm{S}$ no hace A en T' en W', y

c) Para todo mundo W" tal que W" es accesible para $\mathrm{S}$ desde W en T y S no hace A en T' en W, existe un mundo W' tal que

1) W' es accesible para $S$ en $W$ en $T$

2) S hace A en T' en W'

3) El valor deóntico para $\mathrm{S}$ en $\mathrm{T}$ de $\mathrm{W}$ " no es mayor que el valor deóntico para $\mathrm{S}$ en $\mathrm{T}^{\prime}$ de $\mathrm{W}$ ' 
Puesto en términos muy simples, diríamos que un comportamiento es moralmente obligatorio cuando, conforme al sistema moral manejado, su realización es superior (tiene más valor deóntico) que su no realización, vistas todas las alternativas empíricamente posibles, mientras que algo está moralmente permitido cuando su realización no es peor (no tiene menor valor deóntico) que su no realización, vistas también todas las alternativas.

De acuerdo con estos conceptos, puede concluirse que la posición de los autores referidos es correcta, puesto que desde una perspectiva conceptual, en situaciones de equivalencia entre alternativas, cualquier curso de acción de entre las mutuamente incompatibles y del mismo valor que se elija está moralmente permitida, existiendo, eso sí, una obligación de llevar a ejecución alguna de ellas. Ello es así porque, en situaciones de ese tipo, sea cual sea la opción escogida de entre las conflictivas, se dará lugar a una situación respecto de la cual no existe ninguna alternativa deónticamente mejor; esto es, ninguna situación en la que se escoja otra alternativa será mejor, puesto que, como son de valor equivalente, a lo sumo serán del mismo valor. De ese modo, la situación se ajusta a la definición de 'permisión moral', pero no a la de 'obligación moral', puesto que con ninguna de las alternativas se da lugar a una situación deónticamente mejor que con las demás. Lo que sí que existe, como bien sostienen estos autores, es una obligación moral de realizar alguno de los comportamientos moralmente equivalentes, puesto que en este caso, cualquier situación en la que se ejecuta cualquiera de las dos obligaciones prima facie en conflicto resulta moralmente mejor que cualquier otra situación en la que ninguna de ellas es cumplida. Usando un ejemplo: puede que resulte equivalente y por ende moralmente neutra la elección entre salvar a A y no salvar a B, o salvar a B y no salvar a A (siendo imposible salvar a ambos), y en consecuencia cualquiera de esas dos alternativas está moralmente permitida (en tanto que no existe una alternativa mejor, sea cual sea la elección tomada), pero en todo caso habría una obligación moral de salvar a uno de los dos, en tanto que 
cualquier alternativa en que eso ocurre es deónticamente mejor a la alternativa de no salvar a ninguno.

\section{La incomparabilidad}

Un segundo tipo de contexto que parece apropiado para dar lugar a la aparición de dilemas morales es el de las situaciones de incomparabilidad. De forma similar a lo que ha ocurrido con la discusión sobre los dilemas morales, también se ha desarrollado una interesante y compleja discusión filosófica acerca de la incomparabilidad, si bien en este debate suele preferirse hacer uso del término 'inconmensurabilidad'. Si aquí uso la expresión 'incomparabilidad' es porque, siguiendo la línea de algunos autores, considero que se trata de dos situaciones diferenciables y que la más "interesante", por lo que a las consecuencias para el razonamiento práctico se refiere, es la de incomparabilidad. Es relativamente habitual, sin embargo, usar ambos términos como sinónimos ${ }^{39}$, normalmente en el sentido de 'incomparabilidad'.

Como se acaba de apuntar, el debate filosófico sobre la incomparabilidad es rico y complejo, por lo que aquí me limitaré a unas pequeñísimas pinceladas ${ }^{40}$, tomando como referencia la obra de Ruth

${ }^{39}$ Como es el caso de J. Raz en RAZ, J. (1986), The Morality of Freedom, Oxford, Clarendon Press, p. 322; también E. Millgram en MILLGRAM, E. (1997), "Incommensurability and Practical Reasoning", p. 151, en CHANG, R. (1997) (ed.), Incommensurability, Incomparability and Practical Reason, Cambridge (Mass.), Harvard University Press, pp.151-169; o S. Lukes en LUKES, S. (1997), p. 184, en "Comparing the Incomparable: Trade-offs and Sacrifices", en CHANG, R. (1997) (ed.), Incommensurability... (cit.), pp. 184-195.

${ }^{40}$ Con algo más de detenimiento, y examinando las conexiones entre la incomparabilidad y las situaciones de dilema, puede verse MARTíNEZ ZORRILLA, D. (2004), Conflictos... (cit.), Cap. IV secc. 2.2. 
Chang ${ }^{41}$. Esta autora distingue entre los conceptos de 'inconmensurabilidad' e 'incomparabilidad'. La inconmensurabilidad consistiría simplemente en la imposibilidad de valorar dos o más elementos (objetos, situaciones, alternativas de acción, etc.) conforme a una única escala de valor de tipo cardinal. Por ejemplo, la amistad que nos une con distintas personas parece un caso de inconmensurabilidad (sería extraño que afirmásemos, por ejemplo, que con A nos une una amistad de 8,3 puntos en una escala del 0 al 10, mientras que con B nos une una amistad de 6,7 puntos, en la misma escala). Parece haber muchísimas situaciones de inconmensurabilidad, que por otro lado no parecen resultar habitualmente muy problemáticas, ya que en la mayoría de casos no impiden realizar juicios de valor comparativos (por ejemplo, a pesar de no contar con una escala numérica del valor 'amistad', podemos afirmar que con $\mathrm{A}$ nos une una amistad más estrecha que con $\mathrm{B}$, o que nuestra amistad tanto con A como con B es igual de intensa). Dicho en otros términos, como en ocasiones se afirma, la inconmensurabilidad impide realizar comparaciones en una escala cardinal, pero no impide las comparaciones en una escala ordinal (mejor, peor, del mismo valor que).

Por su parte, la incomparabilidad supone la imposibilidad de establecer una relación comparativa afirmativa entre dos o más elementos (objetos, situaciones, alternativas de acción, etc.) ${ }^{42}$. Usualmente se considera que las relaciones comparativas (evaluativas) son tres: 'mejor que', 'peor que' y 'del mismo valor que' (lo que Chang denomina como 'tesis tricotómica'). En consecuencia, la incomparabilidad supone que dados dos elementos A y B, es falso que A sea mejor que B, que A sea

${ }^{41}$ Vid. CHANG, R. (1997), "Introduction”, en CHANG, R. (ed.) Incommensurability... (cit.), pp. 1-34, y CHANG, R. (1998), “Comparison and the Justification of Choice”, en University of Pennsylvania Law Review, vol. 146, n 5, pp. 1487-1528.

${ }^{42}$ CHANG, R. (1997), "Introduction” (cit.), pp. 4-5. 
peor que B, y que A y B sean del mismo valor. Como se verá fácilmente, los conceptos de 'incomparabilidad' e 'inconmensurabilidad' son fácilmente distinguibles, y mientras que la incomparabilidad implica asimismo la inconmensurabilidad, lo contrario no ocurre. También resulta fácil ver que la incomparabilidad tiene (o puede tener) consecuencias de mayor alcance para el razonamiento práctico, sobre todo si, como hacen Chang y de hecho la mayoría de los autores ${ }^{43}$, se comparte una concepción 'comparativista' de la razón práctica, según la cual toda elección en este ámbito para estar justificada requiere descansar sobre un juicio comparativo entre las alternativas de acción en juego. Si tal comparación es imposible, se sigue que cualquier elección estará injustificada.

Como atinadamente recuerda Chang, hay que tener muy presente que todo juicio comparativo requiere de un 'valor de cobertura' (covering value) respecto del cual dicho juicio cobra sentido. No son posibles las comparaciones en abstracto o simpliciter. Si por ejemplo queremos comparar un cuadro de Rembrandt con otro de Picasso, podemos hacerlo atendiendo a distintos valores de cobertura, y no sólo cuantitativos (como por ejemplo comparando las dimensiones), sino evaluativos o valorativos, como por ejemplo el nivel de perfección en la ejecución pictórica o la originalidad de la obra. Es perfectamente posible que las ordenaciones resulten incompatibles atendiendo a los distintos valores de cobertura relevantes: si, por ejemplo, se trata de escoger qué destino es mejor para

\footnotetext{
${ }^{43}$ Algunos autores se desmarcan implícita o explícitamente de esta concepción. A modo de ejemplo, vid. RAZ, J. (1997), "Incommensurability and Agency”, en CHANG, R. (1997) (ed.), Incommensurability... (cit.), pp. 110-128; GRIFFIN, J. (1997), "Incommensurability: What's the Problem?", en CHANG, R. (1997) (ed.), Incommensurability... (cit.), pp. 3551; STOCKER, M. (1997), “Abstract and Concrete Value: Plurality, Conflict, and Maximization”, en CHANG, R. (1997) (ed.), Incommensurability... (cit.), pp. 196-214; o ANDERSON, E. (1997), "Practical Reason and Inconmensurable Goods", en CHANG, R. (1997) (ed.), Incommensurability... (cit.), pp. 90-109.
} 
ubicar nuestra residencia, y hemos de escoger entre vivir en Oxford, donde siempre llueve, o vivir en las Bahamas, la ordenación de estas alternativas con toda seguridad será distinta en función de si el valor de cobertura es la bondad del clima o si lo es la posibilidad de proyección de nuestra carrera universitaria, por poner dos ejemplos. Esta disparidad de ordenaciones en función de los valores de cobertura utilizados es un dato muy relevante, como intentaré mostrar más adelante.

Como se dijo anteriormente, una situación de incomparabilidad sería aquélla en la que no puede establecerse ninguna relación comparativa afirmativa, o, dicho en otros términos, en la que resulta falso (o al menos no verdadero, para dar cabida, como en ocasiones de hace, a la indeterminación del valor de verdad) que $\mathrm{A}$ es mejor que $\mathrm{B}$, que $\mathrm{A}$ es peor que B, y que A y B son del mismo valor. No entraré en la discusión de los argumentos dados a favor y en contra de la posibilidad de las situaciones de incomparabilidad, aunque sí me referiré a la original y sugerente posición de Chang sobre el tema, por considerarlo relevante para lo que se dirá posteriormente acerca de las consecuencias de la incomparabilidad para el razonamiento práctico. Chang afirma que no existen auténticas situaciones de incomparabilidad, sino que lo que ocurre es que al menos en muchos de los casos en los que así lo parece, entre los elementos se plantea otro tipo de relación comparativa "no tricotómica",

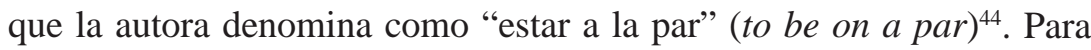
explicar en qué consiste tal relación, Chang sostiene que deben distinguirse dos aspectos o dimensiones comparativas: a) por una parte, la diferencia entre dos o más elementos puede ser 'cero' o 'no-cero', mientras que por otro lado ésta puede estar 'sesgada' (biased) a favor de alguno de los elementos, o bien 'no sesgada' (unbiased). Las relaciones comparativas clásicas pueden ser vistas de acuerdo con estas dimensiones: a) 'A es

${ }^{44}$ Vid. CHANG, R. (1997), “Introduction” (cit.), pp. 25-27. 
mejor que B': existe una diferencia no-cero entre A y B y sesgada a favor de A; b) 'A es peor que B': existe una diferencia no-cero entre A y B y sesgada a favor de B; c) 'A y B son del mismo valor': existe una diferencia cero y no-sesgada entre A y B. Según Chang, todavía es posible otra posibilidad: 'A y B están a la par': hay una diferencia no-cero y no sesgada entre $\mathrm{A} \mathrm{y}^{45}$. Así, las situaciones en las que podría decirse que hay incomparabilidad (como, por hipótesis, y usando un ejemplo de $\mathrm{Raz}^{46}$, la situación en la que una persona debe elegir entre cursar estudios de derecho o seguir su carrera de clarinetista, existiendo en ambos casos unas posibilidades de éxito y de realización personal relativamente iguales), en realidad las distintas opciones están a la par, que es otro tipo de relación comparativa.

Como ya dije en otro lugar, creo que la posición de Chang entremezcla de manera inadecuada las dimensiones cuantitativa y evaluativa de las comparaciones. Que la diferencia entre dos elementos sea cero o no-cero, depende de una comparación que toma como base un criterio comparativo de tipo cuantitativo, esto es, descriptivo o no evaluativo. Conforme a un criterio de este tipo, las posibles relaciones comparativas son 'mayor que', 'menor que' e 'igual que'. Las relaciones comparativas 'mejor que', 'peor que' e 'indiferente' (i.e. del mismo valor que) son en cambio evaluativas o valorativas. Éstas últimas son las relevantes en el ámbito en el que nos estamos moviendo, y para ellas la única dimensión relevante de las dos que maneja Chang es la que distingue entre diferencias 'sesgadas' y 'no sesgadas' ${ }^{47}$. Y desde el punto de vista

\footnotetext{
${ }^{45}$ En realidad, existe todavía otra posibilidad lógica de la que la autora nada dice: una diferencia cero y a la vez sesgada a favor de alguno de los elementos.

${ }^{46}$ En RAZ, J. (1986), The Morality... (cit.).

${ }^{47}$ Aunque se trata de otra discusión, aquí se plantea la importante cuestión de en qué medida la dimensión evaluativa depende la cuantitativa; esto es, si el hecho de considerar un elemento como mejor, peor, etc. que otro depende necesariamente o no, y en qué medida, o bajo qué circunstancias, etc., de diferencias en aspectos descriptivos cuantificables.
} 
de la dimensión comparativa evaluativa respecto de un valor de cobertura determinado, sólo parecen ser posibles las tres relaciones propias de la llamada 'tesis tricotómica': o bien un elemento es mejor que el otro, o bien son todos del mismo valor.

Si la 'tesis tricotómica' es adecuada, parece cobrar plausibilidad la tesis de que en ocasiones se plantean situaciones de incomparabilidad. Pero ello, en mi opinión, sería una conclusión precipitada. Tomemos como base un ejemplo de la propia Chang, en el que debe elegirse al mejor de entre dos candidatos para ocupar una plaza de profesor de filosofía. Uno de esos candidatos es algo mejor que el otro en aspectos como la claridad o la profundidad de análisis, mientras que el otro es algo mejor en aspectos como la originalidad de su pensamiento y el nivel general de conocimiento del pensamiento filosófico. Supongamos que el único valor de cobertura relevante para realizar la comparación que fundamenta la decisión es el de 'talento filosófico'. En este contexto, parece (o así lo consideraremos, por hipótesis) que ninguno de los dos aspirantes es mejor que el otro, pero al mismo tiempo tampoco parecen ser totalmente indiferentes, puesto que cada uno de ellos destaca más que el otro en aspectos que resultan importantes y valiosos para el cargo al que aspiran. Algunos dirían, pues, que como ninguno es mejor que el otro ni son del mismo valor, existe incomparabilidad (para Chang, en cambio, estarían a la par). Sin embargo, considero que hay otro modo más plausible de concebir la situación, que no implica ni sostener la incomparabilidad ni tampoco renunciar a la tesis tricotómica.

Si observamos un valor de cobertura como el de 'talento filosófico', sin duda veremos que se trata de un valor complejo en el que intervienen o para el cual contribuyen otros elementos más simples, como por ejemplo el de la claridad de pensamiento, la profundidad del análisis, el grado de conocimientos filosóficos, o la originalidad del pensamiento, entre otros. Dentro de cada uno de estos elementos más simples, parece 
que es posible establecer una relación comparativa tricotómica: respecto a la profundidad de análisis, por ejemplo, o A es mejor que B, o B es mejor que A, o A y B son equivalentes. Es perfectamente posible, por otro lado, que la ordenación sea la inversa cuando se contempla otro de estos elementos o valores de cobertura más simples. Mi hipótesis, que en cierto modo sigue la línea de autores como Kornhauser ${ }^{48}$ o Seung y Bonevac $^{49}$, es que los supuestos en que parece existir incomparabilidad lo que ocurre es que tenemos un valor de cobertura complejo en el que distintos elementos contribuyentes establecen ordenaciones incompatibles, conjuntamente con el hecho de que carecemos de procedimientos, esquemas o metacriterios que nos permitan "integrar" estos distintos elementos a fin de poder realizar una ordenación definitiva, que tenga en cuenta todos los aspectos relevantes (en este ejemplo, todos los elementos que formarían parte del valor 'talento filosófico'). Cuando uno de los candidatos es mejor que el otro en todos esos aspectos, o en la mayoría de ellos en un grado suficiente, no existen dudas acerca de la ordenación comparativa que ha de fundamentar la decisión, pero si las ordenaciones son contrapuestas y ninguno de los dos destaca de manera clara en la mayoría de aspectos, podemos encontrarnos en una situación de cierta perplejidad porque carecemos que mecanismos, metacriterios o esquemas claros que nos permitan realizar una ordenación definitiva. Por esa razón, aunque estrictamente hablando no existiría incomparabilidad en el sentido arriba indicado, se trataría asimismo de una situación de indeterminación respecto de la que tampoco habría demasiados problemas para denominarla 'incomparabilidad'. Y lo que es más relevante para el tema tratado en el presente trabajo, se trataría de situaciones de dilema, puesto que si en el ámbito del discurso moral se plantea una situación de este tipo (a modo

${ }^{48}$ Vid. KORNHAUSER, L.A. (1998), “No Best Answer?”, en University of Pennsylvania Law Review, vol 146, n 5, pp. 1599-1637.

${ }^{49}$ Vid. SEUNG, T.K. y BONEVAC, D. (1992), "Plural Values and Indeterminate Rankings", en Ethics 102, pp. 799-813. 
de ejemplo, cuando tratamos de decidir si cumplimos o no una promesa importante pero que al tiempo afecta de manera considerable a intereses de terceros), tampoco podríamos determinar la obligación definitiva de entre las diversas obligaciones prima facie en conflicto.

Con todo, creo que puede decirse que toda esta discusión tiene únicamente relevancia teórica (lo que no es poco, por otro lado), pero no consecuencias prácticas, en el sentido de que las consecuencias normativas puedan ser distintas en función de si existe o no "auténtica" incomparabilidad. Para algunos autores (los que pueden denominarse, con Chang, como 'comparativistas'), determinar si existe o no incomparabilidad es de suma importancia, puesto que de ello dependerá la posibilidad de tomar o no decisiones justificadas. Si se dice que no pueden darse casos de auténtica incomparabilidad, aun cuando se introduzcan nuevas relaciones comparativas (como 'estar a la par'), se salvaría, por así decirlo, la racionalidad del discurso práctico. Pero si atendemos a los conceptos de obligación y permisión moral de Zimmerman, en los que nos hemos basado anteriormente, podremos comprobar que en realidad las consecuencias serían idénticas en todos estos posibles supuestos: tanto si se trata de "auténtica" incomparabilidad, como si se trata de falta de procedimientos para la integración de valores de cobertura complejos que permitan elegir entre ordenaciones contrapuestas, como si se trata de supuestos de comparabilidad "no tricotómica", la respuesta sería que cada uno de los cursos de acción incompatibles está permitido (o, dicho de otro modo, que existe una obligación moral de realizar alguno de los comportamientos prima facie obligatorios incompatibles). Como se dijo, si consideramos que un comportamiento está moralmente permitido si no existe otra alternativa deónticamente mejor, no se puede sino concluir que eso es lo que ocurre en cualquiera de los distintos supuestos aquí referidos, de modo que la respuesta es la misma que en los supuestos de equivalencia. De hecho, puede afirmarse que esa será la respuesta en todos los casos de dilema, con independencia de cuál sea su fuente. 
VI.

Si lo que se ha dicho hasta aquí es correcto, no debe sino concluirse que en los casos de dilema, siempre y cuando éstos sean posibles y con independencia de las razones que den lugar a tales situaciones, existe discrecionalidad por parte del agente para elegir qué curso de acción tomar, sin que se pueda decir que hay una "respuesta correcta", o que la decisión tomada sea, estrictamente hablando, incorrecta. Pero esto, aunque pueda considerarse acertado desde el punto de vista teórico, no está exento de problemas, tanto en el ámbito moral como en el jurídico. Tomemos el ejemplo, por hipótesis dilemático, del médico que sólo puede tratar a uno de entre dos pacientes, llamémosles A y B, que de modo irremediable fallecerán si no son tratados. El razonamiento del médico podría ser el siguiente: "como sólo tengo recursos materiales y temporales para salvaros a uno de vosotros, y se trata de una situación de equivalencia en la que cualquier decisión es correcta, elijo salvar a A". Frente a esto, a muchos no nos parecería extraño ni descabellado que $\mathrm{B}$ respondiera del siguiente modo: "Pues si realmente da lo mismo salvar a uno que a otro, ¿por qué no me salva a mí? ¿Qué es lo que le lleva a salvar a A en lugar de hacer lo propio conmigo? Si no existe ninguna razón que fundamente su decisión de preferir a A, su elección es totalmente arbitraria, y usted será en último término el responsable de mi muerte, porque yo merezco ser salvado tanto como A". Algo similar ocurriría en el ámbito jurídico, en el contexto de un conflicto constitucional en el que las pretensiones de ambas partes pueden considerarse prima facie amparadas por derechos constitucionales y en el contexto moral la situación sea de dilema. Si el juez elige amparar la pretensión de A reconociendo que del mismo modo podría haber amparado la de B, B podría replicar: "Pues si realmente da lo mismo, ¿por qué no ampara mi pretensión? A no tiene ninguna razón para pretender que su punto de vista merezca más protección que el mío, así que ¿en qué se fundamenta que se me sacrifique un derecho para proteger el de la otra parte, cuya pretensión no merece mayor protección jurídica que la mía?". 
En consecuencia, parece que aun a pesar de que se considerase correcto, desde la perspectiva del sistema normativo manejado, optar por cualquiera de las alternativas del conflicto, esta solución no resulta demasiado satisfactoria desde el punto de vista de sus consecuencias prácticas. ¿Pero existiría alguna otra manera de enfrentarse a esta situación que resultase más satisfactoria (o menos insatisfactoria) que la de elegir discrecionalmente? Quizás, al menos para determinados contextos, sí que exista. J.M. Taurek ${ }^{50}$, en un artículo de 1977 donde pone en tela de juicio la concepción utilitarista conforme a la cual "los números cuentan" o, dicho de otro modo, que en un contexto en el que no puede evitarse un mal de la misma o similar entidad (por ejemplo, la muerte) a todos los afectados, hay que optar por salvar al mayor número, sostiene que no sería moralmente incorrecto optar por salvar al menor número ${ }^{51}$. Lo interesante de su punto de vista para lo que ahora nos interesa es que afirma que probablemente lo más adecuado en estas situaciones sea utilizar algún tipo de mecanismo aleatorio (el autor pone el ejemplo de lanzar la moneda al aire, pero podría ser cualquier otro) para tomar la decisión, porque de ese modo todos los afectados cuentan con exactamente las mismas probabilidades y así se expresa mejor la idea (de tradición kantiana) de que todas las personas son merecedoras de igual respeto, dignidad y derechos $^{52}$. En el ejemplo usado por Taurek, si se opta por salvar al mayor

${ }^{50}$ En TAUREK, J.M. (1977), “Should the Numbers Count?”, en Philosophy and Public Affairs vol. 6, n. 4 (verano 1977), pp. 293-316.

${ }^{51}$ El ejemplo que utiliza es el siguiente: existen seis personas enfermas, que fallecerán si no se les suministra determinado medicamento. Desgraciadamente, la cantidad de medicamento disponible no permite salvar a todos, porque cinco de ellos necesitarían 1/5 parte del total, mientras que otro enfermo necesitaría la dosis completa. Por tanto, hay que elegir entre salvar a cinco y dejar que el más enfermo muera, o salvar al que necesita toda la dosis y dejar que los otros cinco fallezcan. Taurek sostiene que no existe ninguna razón moral por la que exista una obligación de salvar a estos cinco a expensas de la otra persona (aunque sería una opción moralmente legítima).

${ }^{52}$ Vid. TAUREK, J.M. (1977), “Should...” (cit.), pp. 303 у 306. 
número, estas personas que forman parte del conjunto se verían ilegítimamente favorecidas por el dato contingente de formar parte del conjunto más numeroso, mientras que el menor número se ve (conceptualmente) sacrificado por la simple razón (moralmente irrelevante) de formar parte del conjunto menor. Tratar a todos con la misma consideración y respeto implicaría, para Taurek, conceder a todos las mismas posibilidades, en tanto que seres humanos merecedores de igual respeto.

Creo que esta idea resulta interesante como propuesta normativa para dar respuesta a las situaciones de dilema, al menos para aquéllas en las que se vean afectados los intereses de distintas personas (situación que, partiendo de la hipótesis de que los dilemas son posibles, no sería inusual). En lugar de que el agente tome una decisión entre las distintas alternativas moralmente permitidas, lo que, parece que fundadamente, daría pie a las críticas de quien se ve perjudicado por la decisión, quizá sería preferible optar por un mecanismo aleatorio que otorgue las mismas probabilidades a todos los afectados. En el ejemplo del médico que sólo puede tratar a uno de los dos pacientes (por hipótesis, dilema moral por equivalencia de las alternativas), muchos consideraríamos la reacción del paciente no elegido como natural o incluso como justificada. Sin embargo, parece que no diríamos lo mismo ante una reacción de ese tipo cuando la decisión de a quién tratar hubiera sido el resultado del azar, siempre que todos hubiesen tenido las mismas probabilidades. La situación, en mi opinión, es similar a lo que ocurre con las redistribuciones de riqueza derivadas de los juegos de la lotería: si bien muchos diríamos (creo que con razón) que no sería correcto que los poderes públicos decidieran, por sí mismos y sin criterio o razón alguna, enriquecer a determinados ciudadanos, escogidos sin motivo por los propios poderes públicos, pocos piensan que las redistribuciones de riqueza derivadas de la lotería (en ocasiones, muy importantes) son moralmente injustas, a pesar de que en realidad son tan inmerecidas como las anteriores. En la medida en que son el resultado de un juego de azar en los que todos los participantes cuentan con las mismas probabilidades, no parece que nadie sea tratado injustamente. 
A pesar de todo, y aun cuando esta propuesta pudiese considerarse adecuada, parece que sólo resultaría aceptable en el contexto del discurso moral, pero difícilmente lo sería en el jurídico, aun en las situaciones en las que el problema remitiera a un dilema moral. A nadie parece resultarle atractiva la situación en la que un fallo judicial se tomase lanzando una moneda al aire, a pesar de que existiera acuerdo en que la situación fuera de dilema. El ámbito jurídico posee una dimensión institucional que parece resultar incompatible con la decisión por azar. Pero como hemos visto anteriormente, tampoco resulta atractiva la idea de que el órgano decida discrecionalmente en cada caso de dilema que se le presente. Parece pues en el contexto jurídico la respuesta debe buscarse por otra parte. Aunque que pocos negarían que entre los fines del sistema jurídico se encuentra el de promover la justicia, el contexto jurídico es más complejo y está sujeto a una serie de consideraciones importantes (relacionadas con el mantenimiento del orden, la seguridad, la resolución de conflictos, etc., manifestados en su dimensión institucional) que hacen imposible un traslado automático de la solución moralmente correcta o aceptable al ámbito jurídico. Un aspecto que parece relevante del contexto jurídico es la importancia que suele otorgarse al valor de la seguridad jurídica y la posibilidad de predecir (normativamente, no psicológicamente) las decisiones que ésta conlleva. Una propuesta razonable podría ser la de dar prioridad a la seguridad jurídica frente a la justificación moral de la decisión en los casos de dilema. Creo que algunas de las concepciones de la ponderación (las que en otro lugar he denominado 'universalistas'53) apoyan esta idea. En concepciones como la de Alexy, Moreso o Mendonca, el cometido de la ponderación no es otro que la elaboración de reglas que, tomando como relevantes determinadas propiedades, permitan ofrecer una respuesta clara y determinada a las situaciones de conflicto constitucional, y sobre todo, que actúen como reglas, esto es, que permitan

${ }^{53}$ En MARTÍNEZ ZORRILLA, D. (2004), Conflictos... (cit.), cap. III, sec. 2.2.2. 
la operación de subsunción. En situaciones de conflicto constitucional, es claro que la corrección de la respuesta desde una perspectiva valorativa resulta algo deseable, pero si la situación es de dilema, en la que no puede afirmarse que exista una solución preferible a la otra y que cualquiera de ellas estaría moralmente permitida, resulta mejor, a mi modo de ver, estipular una regla que ofrezca una solución clara para todas las situaciones de ese tipo, aun a sabiendas de que tal regla no es mejor que otra que decidiera en sentido contrario, que dejar la situación indeterminada y a expensas de la libre voluntad del decisor o del azar. 